\title{
Models of Contagious Bovine Pleuropneumonia: Evaluation of Two Novel Strains
}

\author{
Tamara Gull ${ }^{1,6}$, Richard A. French ${ }^{2}$, Timothy S. Gorton ${ }^{2,3}$, Thomas G. Burrage ${ }^{4}$, LeonProzesky ${ }^{5}$, \\ Steven J. Geary ${ }^{2,3}$ and L. Garry Adams ${ }^{*}, 1$ \\ ${ }^{1}$ Department of Veterinary Pathobiology, Texas A\&M University, College Station TX 77843, USA \\ ${ }^{2}$ Department of Pathobiology and Veterinary Science, The University of Connecticut, Storrs, CT 06269, USA \\ ${ }^{3}$ Center of Excellence for Vaccine Research, The University of Connecticut, Storrs, CT 06269, USA \\ ${ }^{4}$ Plum Island Animal Disease Center, Agricultural Research Service, U.S. Department of Agriculture, Greenport NY \\ 11944, USA \\ ${ }^{5}$ Department of Paraclinical Sciences, Faculty of Veterinary Science, University of Pretoria, Onderstepoort, South \\ Africa \\ ${ }^{6}$ Department of Pathobiology, Oklahoma State University, 250 McElroy Hall, Stillwater, OK 74074, USA
}

\begin{abstract}
The clinical course of infection following endobronchial inoculation of three strains of Mycoplasma mycoides subspecies mycoides small colony (MmmSC), the causative agent of Contagious Bovine Pleuropneumonia (CBPP), was compared in 15 mixed-breed Bos taurus steers. Two of these strains have not previously been investigated under experimental conditions. The cattle were inoculated endobronchially with MmmSC strain Gladysdale, Ondangwa or Shawawa and monitored for 42 days. Four of five Gladysdale-inoculated cattle developed acute clinical CBPP. Four of five Ondangwa and 3/5 Shawawa-inoculated cattle developed subacute to chronic CBPP without overt clinical signs but had characteristic pathological lesions. MmmSC was isolated from cattle both during the course of infection and at necropsy. Clinical signs and necropsy lesions were consistent within groups. Ondangwa and Shawawa strains of MmmSC may be useful for study of subacute and chronic CBPP, which is more common in natural infection than peracute CBPP. Use of recent strains will enhance study of CBPP host response, pathogen detection and vaccine development.
\end{abstract}

Keywords: CBPP, bovine, mycoplasma, mycoides, mycoides (SC), pulmonary, foreign, animal, disease.

\section{INTRODUCTION}

Contagious Bovine Pleuropneumonia (CBPP) is a pulmonary disease of cattle and water buffalo [1] caused by the bacterium Mycoplasma mycoides subspecies mycoides small colony (MmmSC). CBPP has profound economic effects on the cattle industry in many parts of Africa and, formerly, coastal Europe [2]. CBPP has primarily been a problem in sub-Saharan Africa. The decrease in governmentsponsored vaccination programs in Africa since the 1980s has allowed the disease to spread virtually unchecked in many areas. Several countries previously thought to have eradicated the disease have experienced recurrences in the last two decades $[2,3]$. With the eradication of rinderpest, CBPP has become the major infectious disease of livestock on the African continent. Eradication of CBPP from both the U.S. and Australia was dependent on strict control of cattle movement, herd-scale slaughter and financial remuneration to owners. Such methods are not feasible in many affected

*Address correspondence to this author at the Department of Veterinary Pathobiology, Veterinary Medical Research Building, Room 110A, College of Veterinary Medicine, Texas A\&M University, College Station, TX 77843-4467, USA; Tel: 979.845.9816; Fax: 979.862.1088;

E-mail: gadams@cvm.tamu.edu nations, as they lack the governmental and civil resources to effectively restrict movement and the financial resources to compensate animal owners.

African and modern European isolates of MmmSC tend to cluster, with each comprised of multiple strains $[4,5]$. These clusters appear to have been diverging since 1700 [6]. Differences in reported morbidity and mortality between the African and European strain clusters of MmmSC may be due to the genetic differences between them or to differing husbandry practices. Some African strains may demonstrate up to $90 \%$ morbidity and $70 \%$ mortality, while European strains typically demonstrate a much lower morbidity and minimal mortality. The causative mycoplasma is transmitted by direct contact, and disease may be peracute to chronic. Cattle may shed MmmSC for weeks prior to demonstrating illness or seroconversion. Clinical signs in affected animals include tachypnea, fever, cough, respiratory distress, lethargy, inappetence, weight loss, pleurodynia and death. Although the disease is characterized by respiratory signs, animals develop mycoplasmemia and demonstrate systemic effects. Septic arthritis with joint distention, synovitis and cartilaginous erosions may be seen in younger animals (usually $<6$ months of age). At necropsy, animals with CBPP demonstrate often-unilateral serofibrinous pleuropneumonia 
characterized by severe interstitial edema and fibrosis [1, 7]. The lungs are often reported to have a marbled appearance due to thickening of interlobular septae and color changes. The chronic form of CBPP may occur upon initial infection or following either acute or subacute disease and may result in the development of encapsulated infected sequestrae within pulmonary tissue in up to $80 \%$ of survivors [8]. Sequestrae are believed to be one of the methods by which CBPP is maintained in cattle populations, as animals with sequestrae may periodically shed MmmSC [9]. Inapparently infected cattle are also known to contribute to the maintenance of the disease in populations.

As no curative treatment for CBPP exists, vaccination is the control method of choice at this time. Vaccination strategies have been employed against CBPP for over 100 years. Multiple vaccine formulations have been developed, but all have drawbacks including the induction of undesirable side effects and lack of consistent protective or long-lived immunity [10]. The currently in-use T1/44 attenuated vaccine requires annual revaccination for protection, which is economically and logistically impractical in many affected countries [2]. Investigation is ongoing toward specific-component vaccines for CBPP [11], but efforts are hampered by the expense and logistical difficulty of conducting field trials.

Antimicrobial treatment has been attempted in CBPP, but has not been found to eradicate the mycoplasma despite in vitro sensitivity [12, 13]. However, antimicrobial administration may decrease the numbers of mycoplasma shed by infected cattle $[14,15]$ and thus reduce the chances of disease transmission.

Most in vivo studies of CBPP have used cattle, as it has proven extremely difficult to cause disease in other ruminant species. Mouse models have been used, particularly in vaccine studies, but mice fail to develop the characteristic lesions of CBPP and researchers instead rely on the induction of mycoplasmemia to evaluate response [16, 17]. In vitro models have been developed but are limited in application [18, 19]. Multiple bovine models have been used for infection, including direct contact, blind endotracheal or endobronchial inoculation and nasal inoculation [20-24]. Previous models inoculated various growth media (minced lung, agar, Hayflick medium, etc.) in addition to the bacterial isolate, and bacterial numbers were variable [21, 24-26]. Inocula could not be quantified in models of direct contact infection. Species, breed or type of cattle were frequently not reported, and some studies of natural infection did not report strain type of the organism beyond 'naturally-occurring field strain'. Dose dependence has been cited as a factor in both disease induction and vaccine efficacy [27-29]. Inoculation of cattle by direct routes (nasal or endobronchial) allows accurate determination of both quantity of organism and incubation period. Infection of cattle with field strains of MmmSC exhibiting differing virulence under the same experimental protocol should permit better comparison of the effects of these strains on the bovine host.

The central hypothesis for this study was that more recent African MmmSC field strains cause a milder subacute to chronic CBPP disease phenotype as compared to the severe acute disease phenotype caused by the Australian Gladysdale strain under standardized experimental conditions. The goal of this study was to infect groups of cattle with identical numbers of three different strains of $\mathrm{MmmSC}$ and to compare their clinical responses, death versus recovery, and pathologic lesions at necropsy. This study, which used both high and low-virulence strains, produced both acute and chronic disease phenotypes. Side-by-side evaluation of acute and chronic phenotypes of CBPP infection should facilitate more relevant comparisons between the models that may suggest enhanced avenues of investigation of disease pathogenesis, diagnostic techniques and host immune response.

\section{MATERIALS AND METHODS}

\section{Experimental Animals}

All experimental protocols were approved by the United States Department of Agriculture/ Department of Homeland Security (USDA/DHS) Plum Island Foreign Animal Disease Center (PIADC) Animal Care and Use Committee. Outbred Bos taurus steers between 6-8 months of age were obtained from a commercial source. No animals showed signs of respiratory disease prior to the start of the study. Animals received an initial physical evaluation by a board-certified large animal internist and anthelmintic treatment with ivermectin $(200 \mu \mathrm{g} / \mathrm{kg} \mathrm{SQ})$ before being used for the study. All steers were tested for CBPP antibodies using the complement fixation test (CFT) and were negative. Animals were acclimated to the experimental rooms for three weeks prior to the start of the experiment. They were housed in groups of five in separate rooms of the indoor biocontainment facility at PIADC. Antibiotic-free alfalfa pellets and water were provided ad libitum. Pens were cleaned daily. All animals were observed by experienced husbandry staff daily. Rectal temperatures were taken weekly prior to inoculation and daily thereafter. Temperatures, clinical signs of illness and attitude were recorded on daily observation sheets.

\section{Sample Collection}

Individual steers were restrained in a chute and headgate. Blood samples were obtained via jugular venipuncture. Nasal swabs for culture and polymerase chain reaction (PCR) were obtained using a sterile guarded rayon veterinary uterine culture swab. Prior to inoculation with $\mathrm{MmmSC}$, all cattle underwent endoscopic bronchalveolar lavage (BAL) using a $1.6 \mathrm{~m}$ video colonoscope. The endoscope was introduced through the right nostril and visually guided through the larynx and into the right caudal lung lobe until bronchial size prevented further advancement. Endoscopic photographs were used to verify placement of the endoscope during each sampling procedure. Two $60 \mathrm{ml}$ aliquots of sterile physiologic saline were instilled through a sterile single-use double-guarded aspiration catheter threaded through the biopsy channel of the endoscope. One $2 \mathrm{ml}$ aliquot of recovered fluid was used for culture and PCR of MmmSC. The endoscope was disinfected prior to use on the next animal. Following the sampling of all cattle in a treatment room, the endoscope was sterilized in glutaraldehyde solution. A dedicated endoscope was used in each room. 


\section{MmmSC Strains}

MmmSC Australian field strain (African cluster) Gladysdale (provided by the Animal and Plant Health Inspection Service, USDA/DHS PIADC, Greenport NY) was used for the high-virulence infection model and African field strains Ondangwa and Shawawa (Nokaneng) (provided by Prof. Leon Prozesky, University of Pretoria, Onderstepoort, South Africa) were used for the lower-virulence models. The Gladysdale strain is routinely used in PIADC's Foreign Animal Disease course and reliably produces acute severe CBPP. The Ondangwa strain originates from a natural outbreak in northern Namibia in 1994, and the Shawawa (Nokaneng) strain from a natural outbreak in northwestern Botswana (Ngamiland) in 1995. The Ondangwa and Shawawa strains have not been evaluated experimentally, and neither strain was passaged in vitro prior to use in this study. Strains were grown in Fortified Commercial medium at $37^{\circ} \mathrm{C}$ for 72 hours and were centrifuged for 15 minutes at $12,000 \times \mathrm{g}$ and $4^{\circ} \mathrm{C}$, then resuspended in $2 \mathrm{ml}$ phosphate buffered saline prior to inoculation. Mycoplasmas were enumerated after centrifugation to verify the inoculation dose.

\section{Pulmonary Infection}

One day after the preinoculation sampling, $10^{10} \mathrm{MmmSC}$ suspended in $2 \mathrm{ml}$ phosphate buffered saline (PBS) were inoculated endoscopically in to the right caudal lung lobe. The inoculation catheter was flushed with 10ml PBS. No additional material was instilled. Sampling as described above was conducted at Day 1 post-infection and weekly until the conclusion of the experiment.

\section{Evaluation of Clinical Disease}

Steers were observed daily by animal husbandry staff familiar with clinical signs of CBPP and daily to weekly by a board-certified large animal internist and a veterinary pathologist, depending on clinical signs observed by the husbandry staff. Rectal temperatures and clinical observations were recorded on daily observation sheets.

\section{Necropsy Procedure}

Cattle were humanely euthanized with intravenous pentobarbital per PIADC animal protocols at the time that clinical signs of disease suggested that death was imminent. If clinical disease was inapparent or observed but resolved, animals were euthanized between Days 36-43 post-infection depending on necropsy facility availability. Animals were necropsied immediately after euthanasia by a veterinary pathologist assisted by a large animal internist. A postmortem bronchoalveolar lavage was done using one $60 \mathrm{ml}$ aliquot of physiologic saline and an aspiration catheter after the lungs were removed from the thoracic cavity and samples were handled as described above. All organs were examined and scored for gross pathology. Lung tissue was cultured to verify the presence of MmmSC. Samples of lung, tracheobronchial lymph node and joint, as well as any other grossly abnormal tissues, were fixed in $10 \%$ buffered formalin for histopathology.

\section{Culture of MmmSC}

Aliquots of BAL fluid, nasal swabs and necropsy tissues were plated or swabbed onto Fortified Commercial agar and grown at $37^{\circ} \mathrm{C}$ for a minimum of 72 hours. Subculture was performed if necessary. Colony morphology was evaluated under a dissecting microscope. Representative colonies were selected for evaluation by PCR.

\section{PCR of MmmSC}

One milliliter aliquots of BAL fluid were centrifuged at $12,000 \times \mathrm{g}$ for 15 minutes at $4^{\circ} \mathrm{C}$. The supernatant was removed and the pellet resuspended in $500 \mu \mathrm{L}$ phosphate buffered saline. The samples were boiled for 10 minutes. Next, $1 \mu \mathrm{L}$ of the sample was combined with $0.9 \mu \mathrm{M}$ concentration of each forward and reverse primer, $0.1 \mu \mathrm{M}$ concentration of probe and TaqMan Universal PCR Master Mix in a total volume of $20 \mu \mathrm{L}$. Real-time PCR was performed with this reaction mixture on the SmartCycler I. Reaction conditions included 2 minutes at $50^{\circ} \mathrm{C}$ followed by 10 minutes at $95^{\circ} \mathrm{C}$ and then 40 cycles of 15 seconds at $94^{\circ} \mathrm{C}$ and 60 seconds at $60^{\circ} \mathrm{C}$. The default threshold limit was set at 30 and the optics detection was turned on for the cycle steps at $60^{\circ} \mathrm{C}$. Data were exported to Microsoft Excel for analysis. Primers used were sequence 5'-ATGGACGAAA GTCTGATGAAGCAATGC-3' and 5'-TCTGGTAAGGTA CTGTCAAGATAAAGTCAT-3', and the probe used was sequence 5'-6FAM-ACAACAGAGATTTACAAC-MGBNF Q-3', in which 6FAM is the fluorescent dye 6-carboxyfluorescein and MGBNFQ is a minor groove binding nonfluorescent quencher. This probe targets a unique polymorphism within the MmmSC 16S RNA gene rrnA [30].

\section{Histopathology}

Tissues collected at necropsy and fixed in $10 \%$ buffered formalin were embedded in paraffin and sectioned at $5 \mu \mathrm{m}$ onto glass slides for light microscopy. Slides were stained with hematoxylin and eosin and scored by a veterinary pathologist familiar with CBPP lesions and blinded as to with which strain the steer had been infected.

\section{Electron Microscopy}

Bronchial epithelial tissues collected at sampling time points via biopsy forceps and at necropsy were processed for thin section analysis. Samples were fixed in a solution containing 10\% neutral buffered formalin. After 1-5 days, necropsy tissues were trimmed into $3 \mathrm{~mm} \times 10 \mathrm{~mm}$ strips and fixed with a solution containing $2.5 \%$ glutaraldehyde in $0.1 \mathrm{M}$ cacodylate buffer ( $\mathrm{pH}$ 7.4) for $24 \mathrm{hrs}$. Biopsy samples were processed similarly, but were not trimmed. After rinsing with $0.1 \mathrm{M}$ cacodylate with $10 \%$ sucrose, the tissue was post-fixed with $1 \%$ osmium tetroxide and $1.5 \%$ potassium ferricyanide for $1 \mathrm{hr}$ at $4^{\circ} \mathrm{C}$. The tissues were stained en bloc with $2 \%$ aqueous uranyl acetate, dehydrated with an acetone series and embedded in Spurr's resin. Ultrathin sections were cut with a diamond knife and contrasted with $2 \%$ methanolicuranyl acetate and Reynolds lead citrate. Images were captured with a Hitachi T-7600 electron microscope operating at $80 \mathrm{kV}$ with an AMT digital camera. Transmission electron microscopy was performed by an experienced electron microscopist.

\section{Serology}

Serum samples collected from the cattle infected with strains Ondangwa and Shawawa were tested by the complement fixation test in the USDA APHIS laboratory at 
Days 0, 24 and 42 post-infection. Cattle infected with strain Gladysdale, which were infected at a different time than the Ondangwa and Shawawa-infected animals, were not tested beyond Day 0, but animals endobronchially infected with this strain typically seroconvert between Days 7-14 postinfection [31] (APHIS, unpublished data).

\section{RESULTS}

\section{Clinical Observations}

Cattle infected with strain Gladysdale were the first to show clinical signs. Two cattle exhibited increased rectal temperature $\left(>39.7^{\circ} \mathrm{C}\right)$ by Day 2 postinoculation, and all but one were febrile by Day 4 (Fig. 1). One steer did not become febrile until Day 6, and this animal never exhibited a fever of above $40.5^{\circ} \mathrm{C}$. In animals that survived, temperatures returned to normal by Day 15 post-infection. In cattle infected with strain Ondangwa, only one animal exhibited a mild increase in temperature $\left(39.2-39.7^{\circ} \mathrm{C}\right)$ between Days 47. One steer infected with strain Shawawa developed fever between $39.2-39.7^{\circ} \mathrm{C}$ on Days 4-6 only.
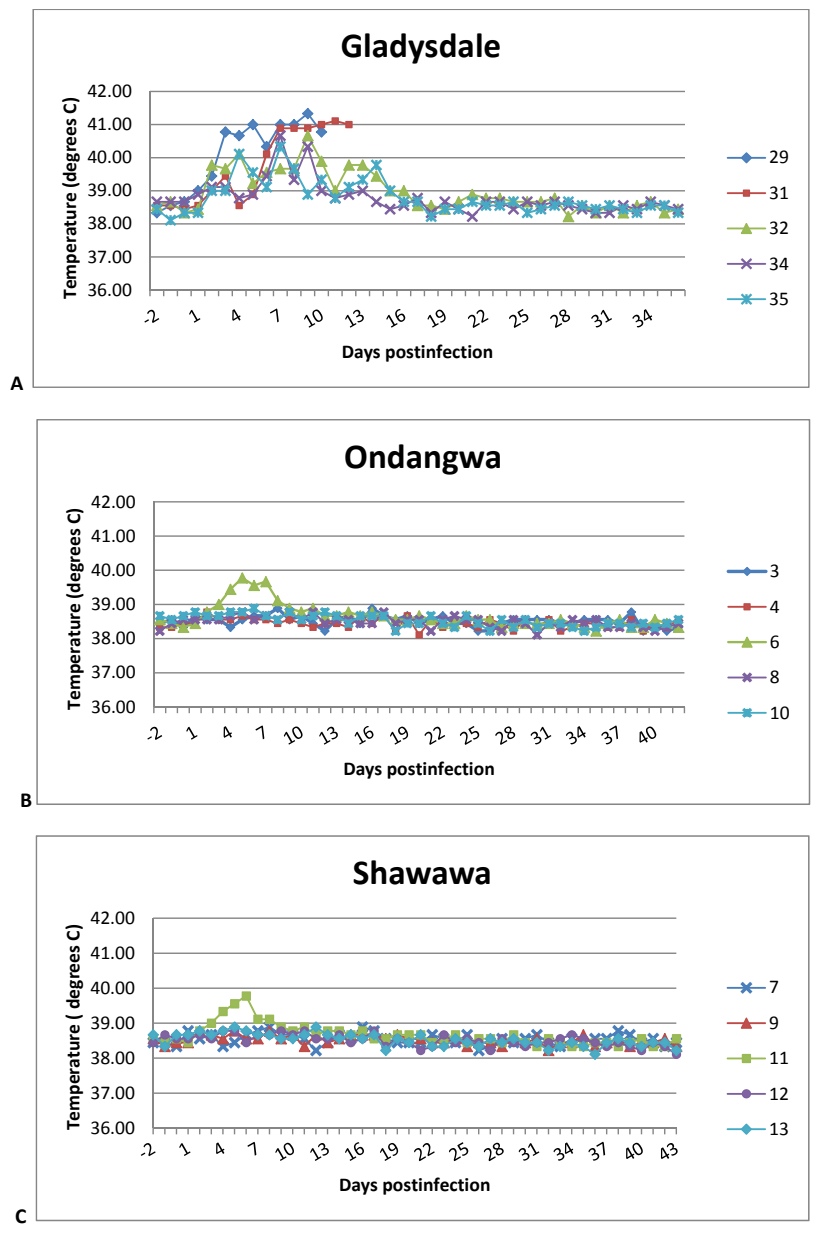

Fig. (1). Rectal temperatures of cattle infected with MmmSC. A. strain Gladysdale; B. strain Ondangwa; C. strain Shawawa. Two Gladysdale-infected cattle were euthanized prior to the conclusion of the experiment due to severe disease.

Two Gladysdale-infected cattle developed tachypnea by Day 3 post-infection. One steer became inappetent on Day
5 , two days after it developed a fever of $>40.5^{\circ} \mathrm{C}$. A second animal became inappetent on Day 6. By Day 7 both of these animals had developed cough and lethargy, and by Day 9 both were standing with abducted elbows and had increased respiratory effort. Unilateral right-sided pleurodynia was evident by Day 9 in both animals. One of these steers developed joint effusion in a tibiotarsal joint on Day 7, and was grade 4/5 lame on the limb (toe-touching lame). One animal demonstrated intermittent open-mouth breathing on Day 9. The two severely-affected animals (\#29 and \#31) became moribund and were euthanized on Days 10 and 13 post-infection respectively.

The three remaining Gladysdale-infected cattle developed tachypnea by Day 8 but never became inappetent. Two exhibited mild unilateral right-sided pleurodynia. No openmouth breathing, increased respiratory effort, altered stance or lameness were observed. Two of the three had an intermittent cough between Days 9-14. One steer had no clinical signs of illness except mild tachypnea and moderate fever that resolved by Day 10.

Two Ondangwa-infected cattle showed mild cough and mild tachypnea between Days 7-12. One Shawawa-infected steer exhibited a cough between Days 6-9. No other clinical signs consistent with CBPP were observed in either the Ondangwa or Shawawa groups. Endoscopic views of the affected areas of lung in all three groups of cattle revealed only mild increases in lung secretions post-inoculation.

\section{Gross Pathology}

Two cattle infected with strain Gladysdale were euthanized when they became moribund. These steers demonstrated severe unilateral pleural effusion; severe, locally extensive unilateral, chronic, fibrinous pleuropneumonia; and moderate, multifocal, coalescing bronchopneumonia with fibrinoeffusive, proliferative pleuritis (Fig. 2, Table 1). These two animals also had moderate, subacute, pericardial effusion; marked, interstitial peritracheal, perivascular, mediastinal and sternal edema; and marked lymphadenomegaly and edema of the tracheobronchial lymph nodes. One of these cattle also had moderate, diffuse, subacute, fibrinous synovitis of the right rear tibiotarsal joint.

The three Gladysdale-infected cattle that were euthanized on Day 36 post-inoculation had varying degrees of pulmonary lesions. Two steers had marked to severe focally extensive, caudal to caudodorsal, chronic pleuropneumonia with sequestrae and focal coalescing mucopurulent to caseous abcesses and severe, focally extensive, chronic, proliferative pleuritis (Figs. 3, 4). The third animal had mild, focal, unilateral, chronic broncho- and interstitial pneumonia. One animal had mild synovial proliferation in a tibiotarsal joint. In all cases, CBPP lesions were on the right side of the pleural cavity and in the caudodorsal right lung, consistent with the site of inoculation. The average gross pathology (Table 2) score for CBPP lung lesions in the Gladysdale group was 4 of 5, and 1.8 of 5 for lesions of the lymph nodes. The lymph nodes of the two nonsurviving cattle had an average score of 4.5 of 5 , and the three surviving cattle (which were euthanized and necropsied more than 20 days after resolution of clinical signs) had lymph node scores of 0 of 5 . 


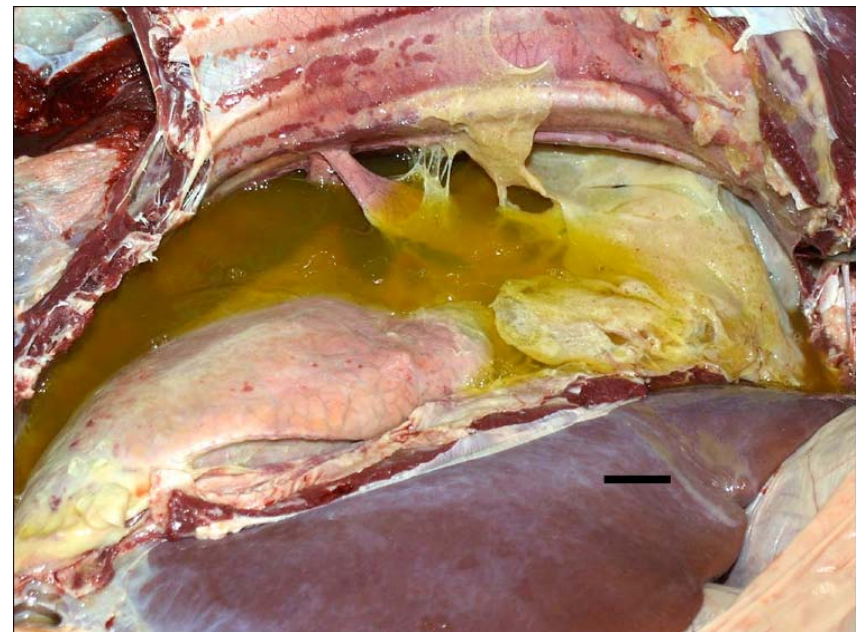

Fig. (2). Gladysdale, 10 days post-infection, right thoracic cavity. Severe, unilateral, chronic, fibrinoeffusive pleuropneumonia. Dorsal is to the left, cranial is to the top of the photo. The thorax was filled with large quantities of a translucent yellow fluid containing strands and sheets of fibrin, the latter of which were adherent to the parietal and visceral pleura. In areas of the caudal dorsal lung, the lobes were adherent to the diaphragm, rib cage and adjacent lobes of the lung. The parietal pleura was multifocally covered by a velvet-like, hyperemic proliferative mesothelium. Bar: $10 \mathrm{~cm}$.

Four of five cattle infected with strain Ondangwa had characteristic gross lesions of CBPP at necropsy on Day 42 post-infection. All lesions attributable to CBPP were located in the right caudal lung consistent with the site of inoculation. Lesions included minimal to severe, focally extensive, unilateral, subacute to chronic, broncho- and interstitial pneumonia. One animal had chronic pleural adhesions attributable to CBPP. No animals had gross lesions of the lymph nodes, and no joint involvement was noted. The average gross lung lesion score for animals infected with strain Ondangwa was 2.8 of 5, and the average lymph node score was 0 of 5 .

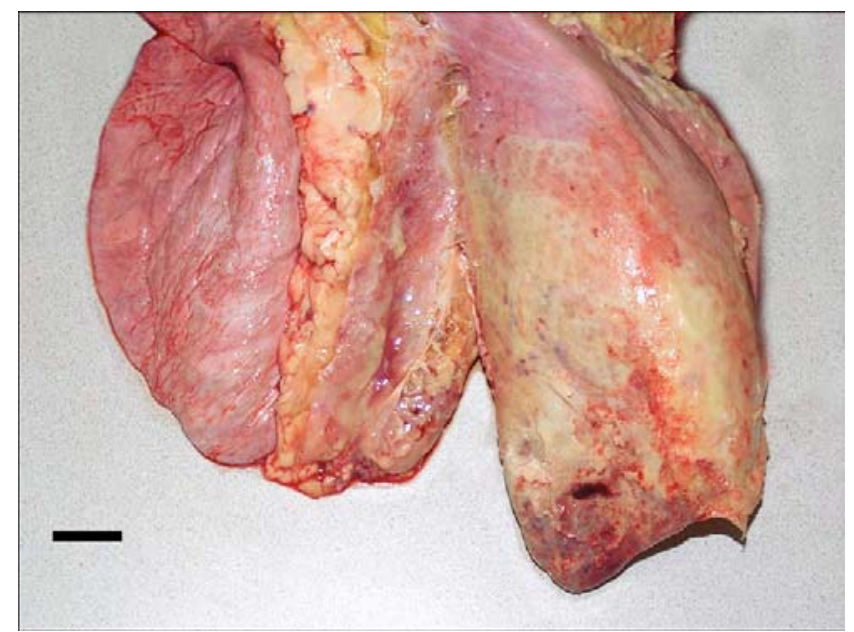

Fig. (3). Gladysdale, 36 days post-infection, lungs and mediastinum. Severe, unilateral chronic fibrinous pleuropneumonia. The right caudal lung was enlarged, firm and covered by a sheet of fibrin. Further cranial in the right lung there is visible widening of the interlobular septae (edema). The left lung was unaffected. Note the widening of the mediastinum, which was due to edema and 5-10X enlargement of the mediastinal lymph nodes. Bar: $10 \mathrm{~cm}$

Table 1. Clinical Results of Infection with MmmSC

\begin{tabular}{|c|c|c|c|c|}
\hline Animal & Strain & Clinical Signs & Outcome & Postmortem \\
\hline 7 & Shawawa & None & survived & None \\
\hline 9 & Shawawa & None & survived & Mild chronic focal BIP \\
\hline 11 & Shawawa & Fever, cough & survived & Mild chronic focal BIP, $\uparrow \mathrm{LN}$ \\
\hline 12 & Shawawa & None & survived & None \\
\hline 13 & Shawawa & None & survived & Mild chronic focal BIP \\
\hline 3 & Ondangwa & Cough, tachypnea & survived & Severe chronic focal BIP, $\uparrow \mathrm{LN}$ \\
\hline 4 & Ondangwa & None & survived & Minimal subacute focal BIP, $\uparrow \mathrm{LN}$ \\
\hline 6 & Ondangwa & Fever, cough, tachypnea & survived & Severe chronic focally extensive BIP, pleural adhesions, $\uparrow \mathrm{LN}$ \\
\hline 8 & Ondangwa & None & survived & $\uparrow \mathrm{LN}$ \\
\hline 10 & Ondangwa & None & survived & Moderate chronic focal BIP, $\uparrow \mathrm{LN}$ \\
\hline 29 & Gladysdale & $\begin{array}{l}\text { Fever, cough, tachypnea, dyspnea, } \\
\text { lameness, inappetence, pleurodynia, open- } \\
\text { mouth breathing }\end{array}$ & Died (Day 10) & $\begin{array}{c}\text { Pleural effusion and adhesions, severe extensive PP and BIP, } \\
\text { synovitis, pericarditis, } \uparrow \mathrm{LN}\end{array}$ \\
\hline 31 & Gladysdale & $\begin{array}{l}\text { Fever, cough, dyspnea, tachypnea, } \\
\text { inappetence, pleurodynia, weight loss }\end{array}$ & Died (Day 13) & $\begin{array}{c}\text { Pleural effusion and adhesions, severe extensive PP and BIP, } \\
\text { pericarditis, } \uparrow \mathrm{LN}\end{array}$ \\
\hline 32 & Gladysdale & Fever, cough, tachypnea, mild pleurodynia & survived & Focal extensive PP and BIP, sequestrum, $\uparrow \mathrm{LN}$ \\
\hline 34 & Gladysdale & Fever, tachypnea & survived & Mild focal PP \\
\hline 35 & Gladysdale & Fever, cough, tachypnea, mild pleurodynia & survived & Focal extensive PP and BIP, sequestrum, $\uparrow \mathrm{LN}$ \\
\hline
\end{tabular}

Fifteen naïve cattle were endobronchially infected with one of three strains of MmmSC and followed through euthanasia and postmortem. Animals annotated as "died" were euthanized in extremis. $\uparrow \mathrm{LN}=$ enlarged tracheobronchial lymph nodes; $\mathrm{BIP}=$ bronchointerstitial pneumonia; $\mathrm{PP}=$ pleuropneumonia 
Table 2. Scoring Algorithm for Gross and Histologic Lesions in Infected Cattle

\begin{tabular}{|c|c|c|c|c|c|}
\hline Gross Pathology Scores & Score & Percentile & Histopathology Scores & Score & Percentile \\
\hline \hline No Gross Lesions & 0 & 0 & No Significant Lesions & 0 & 0 \\
\hline Minimal & 1 & $<5$ & Minimal & 1 & $<5$ \\
\hline Mild & 2 & $5-10$ & Mild & $5-10$ \\
\hline Moderate & 3 & $10-25$ & Moderate & 3 & $10-25$ \\
\hline Marked & 4 & $25-50$ & Marked & 4 & $25-50$ \\
\hline Severe & 5 & $>50$ & Severe & 5 & $>50$ \\
\hline
\end{tabular}

Gross Pathology: Percentile of lung involvement. Histopathology: Percentile of parenchymal involvement in sections of representative lesions. Scoring of gross and histopathology is based on degree of severity. This is generally a matter of \% of parenchymal involvement and is a subjective score. The pathology (distribution, duration, infiltrate/exudate) is consistent in most cases.

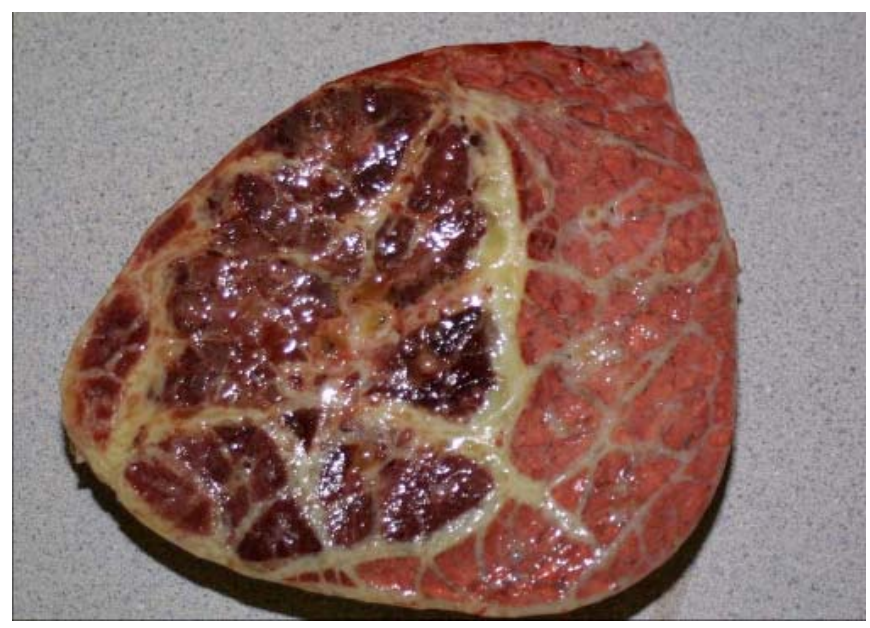

Fig. (4). Gladysdale, 36 days post-infection, sagittal section of right caudal lung. Severe, unilateral, chronic, pleuropneumonia with sequestrum formation. In the right caudal lung was a welldelineated focus of sequestered lung in which the lung parenchyma was dark red, firm and contained foci of necrosis. In the surrounding aerated lung there is marked widening of interlobular septae (edema) and proliferation of fibrous connective tissue (marbling). Tissue section: 12 x 14cm.

Three cattle infected with the Shawawa strain had characteristic gross CBPP lesions at necropsy on Day 43 post-infection, also confined to the right caudal lung at the site of inoculation. These lesions included minimal to mild, focal, unilateral, subacute to chronic broncho- and interstitial pneumonia. No Shawawa-infected cattle had lesions of the lymph nodes or joints. The average gross lung lesion score for cattle infected with strain Shawawa was 0.5 of 5 and the average lymph node score was 0 of 5 .

\section{Histopathology}

Microscopic lesions of the lung in Gladysdale-infected cattle included marked to severe, diffuse, chronic, suppurative bronchopneumonia with bronchial lymphoid hyperplasia in all animals (Fig. 5). Lesions of the tracheobronchial lymph nodes ranged from mild lymphoid hyperplasia to marked, diffuse lymphoid hyperplasia with sinus histiocytosis and edema. Joint lesions in three cattle demonstrated mild to marked diffuse, subacute, proliferative lymphocytic synovitis. Two animals had no microscopic joint involvement. The average histopathology lung lesion score in Gladysdale-infected cattle was $4.6 / 5$ and the average lymph node lesion score was 3.6/5.

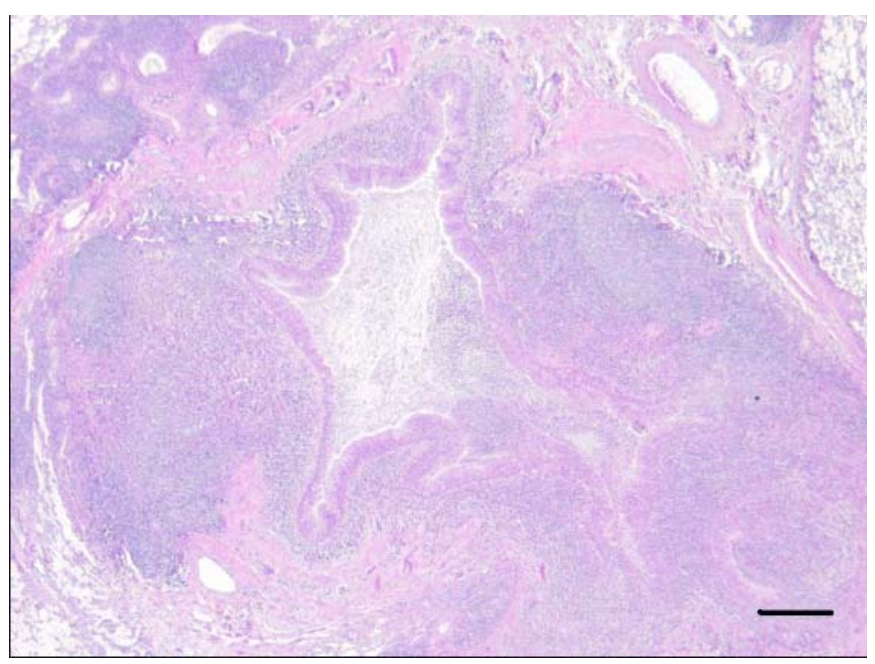

Fig. (5). Gladysdale, 13 days post-infection, right caudal lung bronchiolitis. Severe, chronic, lymphoproliferative bronchopneumonia and bronchiolitis. Magnification: 100x; bar $=1500 \mu \mathrm{m}$.

Cattle infected with MmmSC strain Ondangwa demonstrated moderate to severe, focally-extensive to diffuse, chronic, suppurative interstitial and bronchopneumonia with bronchial lymphoid hyperplasia (Fig. 6). Lesions of the lymph nodes included moderate to marked diffuse, lymphoid hyperplasia of the tracheobronchial lymph nodes. No joint lesions were noted. The average microscopic lung lesion score was $3.2 / 5$ and the average lymph node score was 3.4/5.

Shawawa-infected cattle had lung lesions ranging from mild, diffuse, chronic, bronchial associated lymphoid hyperplasia to marked, diffuse, chronic, interstitial pneumonia with bronchial associated lymphoid hyperplasia to severe, diffuse, chronic bronchiolitis obliterans with severe bronchial associated lymphoid hyperplasia (Fig. 7). Lesions of the lymph nodes in this group were moderate to marked with lymphoid hyperplasia of the tracheobronchial lymph nodes. No joint lesions were noted. The average microscopic lung lesion score in this group was 2.6/5 and the average lymph node score was also 2.6/5. 


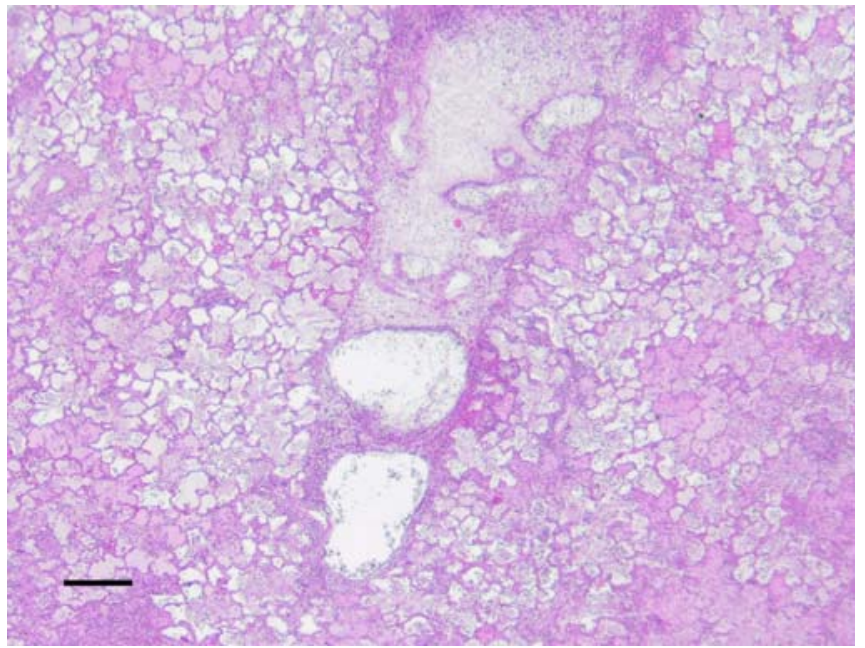

Fig. (6). Ondangwa, 42 days post-infection, pneumonia of right caudal lung. Subacute, sero-fibrinous pneumonia, necrosuppurative and edematous. Magnification: 40X; bar $=1000 \mu \mathrm{m}$.

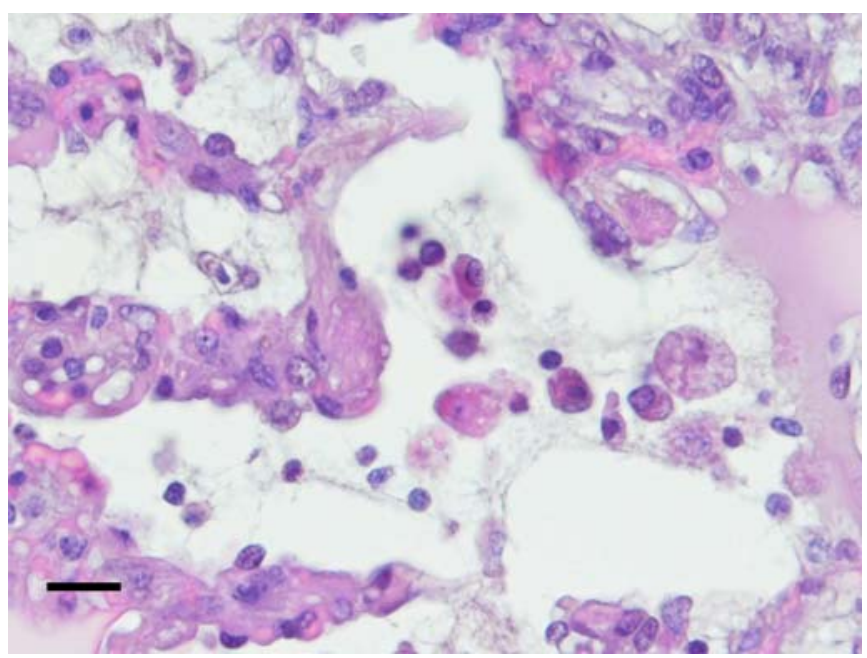

Fig. (7). Shawawa, 43 days post-infection, right caudal lung. Chronic fibrinoeffusive pneumonia with thickened alveolar septae and intra-alveolar lymphocytes and macrophages. Magnification: $1000 \mathrm{X}$; bar $=25 \mu \mathrm{m}$

\section{Transmission Electron Microscopy}

TEM was successful only on Gladysdale necropsy samples. Tissue samples collected via bronchial biopsy contained excessive crush artifact and were unusable. Images of the bronchial tissues collected at necropsy showed the mycoplasma present in large numbers on the epithelial surface, but none were seen within the epithelial cells (Fig. 8). Mycoplasmas were present in high concentrations at the base of the cilia of respiratory epithelial cells. Some epithelial cells showed morphologic changes consistent with apoptosis.

\section{Detection of $\mathrm{MmmSC}$}

Bronchoalveolar lavage and nasal swab samples were culture and PCR negative for MmmSC for all cattle prior to inoculation. Following inoculation, bronchoalveolar lavage samples were positive for MmmSC on culture and/or PCR for all Gladysdale-infected steers at one and two weeks postinoculation. After that time point, culture and/or PCR of
MmmSC were intermittently positive in all animals until necropsy. Nasal swab samples were intermittently positive via culture or PCR throughout the experiment.

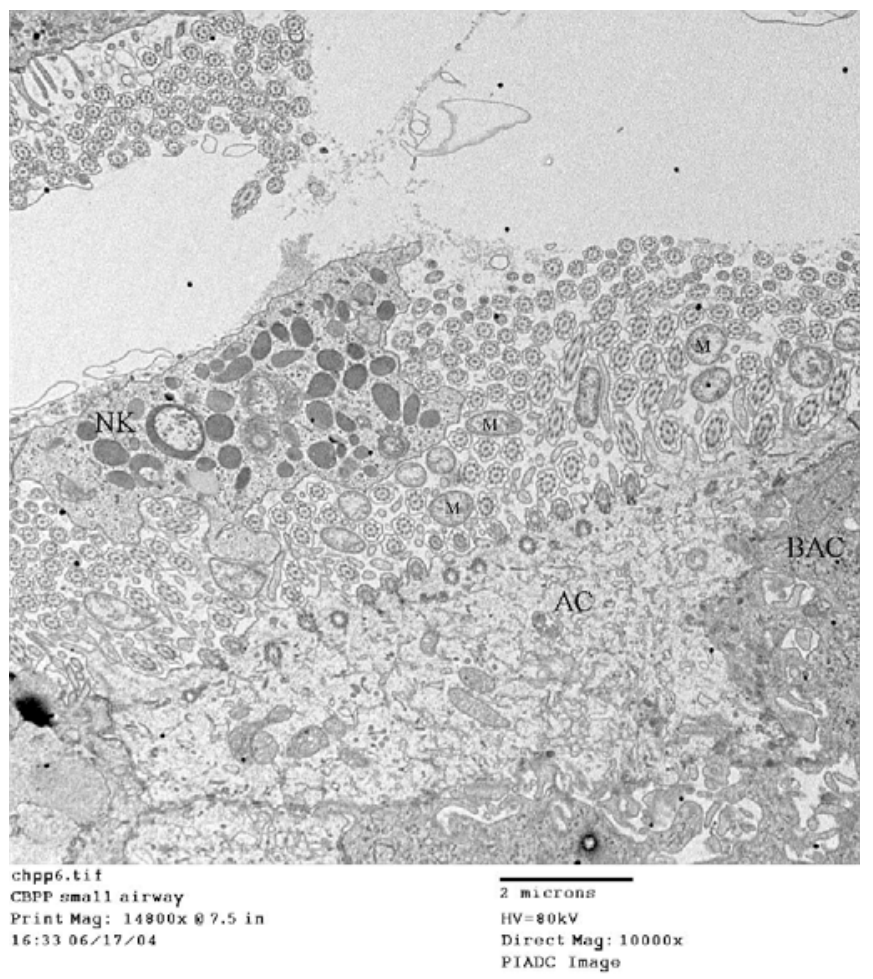

Fig. (8). Gladysdale, 36 days post-infection, transmission electron microscopy, right caudal lung. A natural killer cell adjacent to mycoplasma among the cilia of a bronchial epithelial cell. NK: natural killer cell; M: mycoplasma; BAC: bronchial epithelial cell; AC: apoptotic bronchial epithelial cell. Magnification: 14,800X; bar $=2 \mu \mathrm{m}$.

Bronchoalveolar lavage samples from 4/5 Ondangwainfected cattle were positive for MmmSC via culture or PCR on day 7 and intermittently positive thereafter. Shawawainfected cattle had 2/5 positive bronchoalveolar lavage samples at day 7 with intermittent positives thereafter. In both Ondangwa and Shawawa-infected cattle, nasal swabs were intermittently positive throughout the experiment. Lung lesions from all cattle were culture-positive for MmmSC at necropsy.

\section{Serology}

All steers infected with strains Ondangwa and Shawawa were negative via the complement fixation test at Days 0, 24 and 42 post-infection. Gladysdale-infected animals were not tested by the CFT.

\section{DISCUSSION}

One isolate (Gladysdale) used in this study was selected as it was known to produce severe acute pulmonary disease in Bos taurus cattle during historical Australian field outbreaks and experimental use at PIADC. The Ondangwa and Shawawa isolates are recent African field strains reported to produce moderate-to-severe disease in primarily Bos indicus cattle in natural outbreaks. They were selected for comparison since strain Gladysdale has not been seen in a natural outbreak in more than 60 years. More recent natural 
strains were desired in order to find currently-applicable strains from which immune response data could be collected. While neither modern strain produced disease as severe as the classic Gladysdale strain, the clinical results observed supported their use in that they reliably produced subacute to chronic disease. While the number of animals used was small, the results parallel previous reports as far as morbidity and the percentage of animals demonstrating clinical recovery that still had lesions at necropsy $[8,26]$. Mortality in our models was lower than reported from the field outbreaks. This could be due to many factors including species, breed, nutritional or general health status of the cattle. The natural outbreaks occurred in Bos indicus cattle, while our experimental cattle were Bos taurus. It would have been preferable to obtain Bos indicus cattle; however, PIADC procurement protocols dictate the purchase of cattle from a commercial source and Bos indicus commercial cattle are rare in the northeast United States. While the clinical presentation of each isolate in our study was fairly consistent, it would be anticipated that the use of larger numbers of animals would likely result in a greater variety of clinical disease (acute, subacute or chronic) regardless of strain. However, this experiment may provide a foundational basis for such later investigations.

The onset of clinical disease was rapid in all models compared to natural infection, with the first clinical signs appearing as soon as Day 3 post-inoculation. Steers infected with strain Gladysdale developed more severe clinical disease than did animals infected with either of Ondangwa or Shawawa. Gladysdale-infected cattle also demonstrated higher rectal temperatures, with all animals but one reaching $>40.5^{\circ} \mathrm{C}$ within nine days of inoculation. In comparison, one each of Ondangwa and Shawawa-infected animals reached a maximum of $39.9^{\circ} \mathrm{C}$. The other Ondangwa- and Shawawainfected cattle remained afebrile, although as temperatures were taken only once daily a rapidly spiking and resolving fever could not be ruled out. Fever is an unreliable indicator of CBPP infection, as demonstrated by the three Ondangwa and two Shawawa animals without fever that nonetheless had gross necropsy lesions. Cough appears to be an inconsistent clinical sign, as animals without noticeable cough had lesions of moderate to severe disease at necropsy. Tachypnea was present in most animals starting within the first week post-infection, but no difference was noted between groups.

The spectrum of gross necropsy lesions differed between groups of cattle, with the two Gladysdale-infected cattle requiring early euthanasia demonstrating profound pleural effusion and fibrin formation in addition to extensive lung involvement. The three Gladysdale-infected animals that survived until the end of the experiment had minimal pleural effusion despite two having demonstrated mild pleurodynia earlier in the course of infection. As neither ultrasound examination of the thorax or thoracocentesis was done due to equipment limitations in containment, it is unknown whether these animals had pleural effusion earlier in the course of infection that had resolved by the time of necropsy. Two of these three Gladysdale-infected cattle did develop sequestrae encapsulated with a thick fibrous layer after 36 days of infection. In contrast, Ondangwa- and Shawawa-infected cattle failed to develop classical sequestrae after 42 days of infection despite demonstrating other gross lesions consistent with CBPP. It is unknown whether Ondangwa- or Shawawainfected animals would develop sequestrae given a longer time course, as neither of these strains has been previously experimentally evaluated and there are no published reports of necropsy findings during natural outbreaks ofthese isolates. Another possible explanation is that endobronchial inoculation of MmmSC may result in more chronic disease patterns than natural exposure [32]. However, this does not explain the clinical course and lesions seen in the Gladysdale-infected cattle, which were characteristic of acute severe disease.

It is unlikely that these lesions were caused by the inoculum carrier rather than the MmmSC, as the inoculum consisted of only organism and phosphate-buffered saline, in contrast with other models which have used agar or minced lung tissues [33, 34]. All necropsy lesions were located in the right caudodorsal lung, consistent with the site of inoculation of the MmmSC and MmmSC was isolated from necropsy lung samples. No active lesions were noted in the cranioventral lung as would be expected with bovine respiratory disease complex (BRD) organisms.

Histopathology of lesions from infected cattle demonstrated the full spectrum of classical CBPP lesions. Microscopic pulmonary lesions from Ondangwa- and Shawawa-infected steers were very similar to those from Gladysdale-infected cattle, but the percentage of affected lung was less. All cattle with CBPP lesions, regardless of strain, demonstrated chronic interstitial and suppurative bronchopneumonia with fibrosis. Lung tissue, particularly the airway submucosa, had profound infiltration of lymphocytes and plasma cells with fewer macrophages and occasional neutrophils. Virulence differences between strains appeared to be related to the amount of lung parenchyma each isolate was able to influence rather than the severity of lesions within the affected parenchyma. However, Gladysdale-infected cattle had a greater degree of fibrosis and bronchial lymphoid hyperplasia than did either Ondangwa- or Shawawa-infected cattle, with development of lymphoid follicles within lung parenchyma. The increased fibrosis in Gladysdale-infected animals may reflect that strain's tendency to create sequestrae, while strains Ondangwa and Shawawa were unable to elicit the same response in the host and did not form sequestrae.

Lesions of the tracheobronchial lymph nodes varied between strains, with Gladysdale-infected cattle demonstrating marked lymphoid hyperplasia, edema, and sinus histiocytosis. Lymph node germinal centers in Gladysdale-infected steers that succumbed to CBPP had numerous apoptotic lymphoblastoid cells that were not seen in Gladysdale-infected animals that survived infection or in Ondangwa- or Shawawa-infected animals. Cattle infected with Ondangwa and Shawawa had less prominent lymphoid hyperplasia of tracheobronchial lymph nodes, and edema was absent.

Only Gladysdale-infected cattle had microscopic joint lesions of lymphocytic synovitis, and two of the three cattle with joint lesions were the animals euthanized early in the experiment. Two animals with microscopic synovitis had never been noted to be lame or to have clinical joint effusion. No joint lesions were seen in either Ondangwa- or Shawawainfected cattle; it is unknown if histologic lesions might have 
been present earlier in infection but resolved by Day 42 necropsy.

Transmission electron microscopy of endoscopic bronchial biopsies taken during weekly sampling yielded no useful data due to the small size and excessive crush artifact of the tissue. Images taken from necropsy tissue demonstrated large numbers of mycoplasma colonizing the surface of respiratory epithelial tissue, often predominantly at the base of the cilia. These images do not reveal any evidence of intracellular MmmSC, but the intimacy of contact between the mycoplasma and the epithelial cell could not be definitively determined. Most mycoplasma remain extracellular when infecting a host animal, but certain species are capable of invasion and intracellular survival in non-phagocytic cells [35]. Mycoplasma penetrans has been shown to have fibronectin-binding ability [36] and to alter the host cell cytoskeleton [37], both characteristics of invasive bacteria. Other mycoplasma, most notably $M$. fermentans, are reported to have the ability to fuse with the host cell [38]. No information has been published on whether MmmSC possesses fusogenic capability, although $M$. capricolum, also within the mycoides cluster, is known to be fusogenic [38].

Detection of MmmSC from bronchoalveolar lavage and nasal swab samples was intermittent for all strains used, particularly when no clinical disease was evident. This is consistent with previous reports in which isolation of organism was found to be intermittent in the convalescent phase and in animals with chronic disease. Animals with sequestrae may harbor MmmSC within the lung tissue for months to years with periodic shedding of the organism. Nasal swabs have been previously demonstrated to be of limited usefulness in the diagnosis of CBPP, as they are often negative even when organism can be isolated from lung tissue or secretions [39]. MmmSC was isolated from lung tissue at necropsy from all animals with gross lung lesions. Isolation of other bovine respiratory disease complex organisms was not attempted, and no cattle had characteristic gross lesions of active BRD complex infection.

The negative CFT results in the Ondangwa and Shawawa animals at days 24 and 42 demonstrate the unreliability of that test. The CFT is reported to detect only approximately $70 \%$ of chronic cases of CBPP and often fails to detect asymptomatic animals early in the course of infection and even some symptomatic animals [1, 26, 40-42]. Despite direct inoculation of the organism into the lung, the CFT remained uniformly negative in all Ondangwa and Shawawa animals including those shown to have CBPP lesions and positive cultures at necropsy. The CFT is recommended as a herd level test [43], so the small numbers of animals in our experimental groups may have contributed to the lack of positive CFT results. Additionally, the CFT detects primarily IgM [31]; our animals may have been tested late enough that IgM had been replaced by IgG. Alternatively, we may have tested the cattle too early for seroconversion to occur given the small areas of lung that were affected. The CF tests were performed by experienced staff at the USDA's Animal and Plant Health Inspection Service (APHIS) at PIADC, the United States' official laboratory for CBPP testing, using established positive and negative controls. No additional testing modalities were available, and due to United States rules samples could not be removed from the facility for outside testing. Nonetheless, the CFT is currently the official standard for determining infection per the World Animal Health Organization (OIE) [44], and these results only underscore the need for more sensitive and specific diagnostic options.

In this age of rapid global transportation, there is concern for reintroduction of CBPP into the United States, whether accidental or deliberate. Due to its extended absence from North America, bovine veterinarians and cattle producers are largely unfamiliar with the clinical manifestations of CBPP. This could result in delays in recognition and diagnosis of infected cattle. Also, recognition of CBPP in North America could be hampered because of the similarity between lesions of CBPP and severe Mycoplasma bovis pneumonia [45-47]. Cattle shipping and feedlot management practices in the United States could provide a fertile ground for rapid transmission of CBPP, and delays in diagnosis could prove extremely costly. This underscores the need for further study of CBPP and development of additional means of diagnosis, differentiation from $M$. bovis and continued vaccine development.

\section{CONCLUSIONS}

Direct endobronchial inoculation of $10^{10} \mathrm{MmmSC}$ without agar or other adjuvants remains successful as a model of CBPP infection. We tested this model using three African/Australian cluster strains of $\mathrm{MmmSC}$ reported to have differing virulence. Two of these strains, Ondangwa and Shawawa, have not previously been evaluated in an experimental setting. Results from this study support descriptions of the relative virulence of natural infections involving these isolates: Gladysdale exhibited the greatest virulence, followed by Ondangwa, with Shawawa demonstrating the lowest virulence. Using this infection model, this study generated an acute severe disease group (Gladysdale) and two chronic inapparent disease groups of differing severity (Ondangwa and Shawawa). Morbidity and incidence of necropsy lesions for each inoculated strain were comparable to reports of naturally-occurring disease. The model produced lower mortality rates than those reported in natural infections of each isolate, although the number of cattle infected with each isolate was small and may not represent the full spectrum of disease that could be produced by each isolate.

Advantages of endobronchial inoculation include a known site of infection within the lung that may be repeatedly visualized and sampled as needed, and relatively atraumatic lower respiratory tract sampling in standing cattle. Disease onset was more rapid than that induced by contact infection, compressing the required number of sampling days and allowing all cattle to be sampled as a cohort with a defined time since infection. This model is also adaptable to field conditions: although a videoendoscope was used in this study, a portable light source and visual (rather than video) confirmation of endoscope placement would be equally effective. However, either sampling method requires at least 3 personnel and a means of cattle restraint such as a chute and headgate.

Standardization of inoculum size, placement of inoculum, and time since infection in experimental cattle 
may remove some of the uncertainty present in the studies of natural or experimental in-contact CBPP infections. The development of these three side-by-side CBPP models of differing virulence will also permit more direct comparison of host effects versus inoculum effects. This model, using any of the tested strains, could be applied to discrete subgroups of cattle segregated by species, breed or bovine leukocyte antigen (BoLA) type. The model and data presented in this study should facilitate future analysis of MmmSC strains of differential virulence.

\section{ABBREVIATIONS}

$$
\begin{array}{ll}
\text { APHIS } & \text { Animal and Plant Health Inspection Service } \\
\text { BAL } & =\text { Bronchoalveolar lavage } \\
\text { BoLA } & =\text { Bovine leukocyte antigen } \\
\text { BRD } & =\text { Bovine respiratory disease complex } \\
\mathrm{CBPP} & =\text { Contagious Bovine Pleuropneumonia } \\
\text { DHS } & =\text { Department of Homeland Security } \\
\text { MmmSC } & \text { Mycoplasma mycoides subspecies mycoides } \\
\text { OIE } & =\text { Small colony } \\
\text { PIADC } & =\text { Plum Island Foreign Animal Disease Center } \\
\text { PBS } & =\text { Phosphate buffered saline } \\
\text { PCR } & =\text { Polymerase chain reaction } \\
\text { USDA } & =\text { United States Department of Agriculture }
\end{array}
$$

\section{CONFLICT OF INTEREST}

The authors confirm that this article content has no conflict of interest.

\section{ACKNOWLEDGEMENTS}

Supported by United States Department of Homeland Security/ United States Department of Agriculture grant 58194-02-250 and a U.S. Department of Homeland SecurityNational Center of Excellence for Foreign Animal and Zoonotic Disease (FAZD) Defense grant ONR-N00014-041-0. The authors thank Roberta Pugh, Mindy Barnett, Dr. Luis Rodriguez, Dr. Mary Lou Berninger, Dr. Samia Metwally and Dr. Daniel Rock for technical assistance.

\section{REFERENCES}

[1] Provost A, Perreau P, Bréard A. Contagious bovine pleuropneumonia. Rev Sci Tech 1987; 6(3): 625-79.

[2] Windsor RS. The eradication of contagious bovine pleuropneumonia from south western Africa. A plan for action. Ann N Y Acad Sci 2000; 916: 326-32.

[3] Marobela-Raborokgwe C. Contagious bovine pleuropneumonia in Botswana: experience with control, eradication, prevention and surveillance. Vet Ital 2011; 47(4): 397-405.

[4] Poumarat F, Solsona M. Molecular epidemiology of Mycoplasma mycoides subsp. mycoides biotype Small Colony, the agent of contagious bovine pleuropneumonia. Vet Microbiol 1995; 47(3-4): 305-15.

[5] Vilei EM, Abdo EM, Nicolet J, et al. Genomic and antigenic differences between the European and African/Australian clusters of Mycoplasma mycoides subsp. mycoides SC. Microbiology 2000; 146 (2): 477-486.

[6] Dupuy V, Manso-Silvan L, Barbe V, et al. Evolutionary history of contagious bovine pleuropneumonia using next generation sequencing of Mycoplasma mycoides subsp mycoides "Small Colony". PLoS ONE 2012; 7(10): e46821.
Nicholas RA, Bashiruddin JB. Mycoplasma mycoides subspecies mycoides (small colony variant): the agent of contagious bovine pleuropneumonia and member of the "Mycoplasma mycoides cluster". J Comp Pathol 1995; 113(1): 1-27.

Bygrave A, Moulton J, Shifrine M. Clinical, serological and pathological findings in an outbreak of contagious bovine pleuropneumonia. Bull Epizoot Dis Afr 1968; 16: 21-46.

ter Laak EA. Contagious bovine pleuropneumonia. A review. Vet Q 1992; 14(3): 104-10.

Thiaucourt F, Dedieu L, Maillard JC, et al. Contagious bovine pleuropneumonia vaccines, historic highlights, present situation and hopes. Dev Biol (Basel) 2003; 114: 147-60.

Hubschle OJ, Tjipura-Zaire G, Abusugra I, et al. Experimental field trial with an immunostimulating complex (ISCOM) vaccine against contagious bovine pleuropneumonia. J Vet Med B Infect Dis Vet Public Health 2003; 50(6): 298-303.

Ayling RD, Bisgaard-Frantzen S, March JB, Godinho K, Nicholas RA. Assessing the in vitro effectiveness of antimicrobials against Mycoplasma mycoides subsp. mycoides small-colony type to reduce contagious bovine pleuropneumonia infection. Antimicrob Agents Chemother 2005; 49(12): 5162-5.

Yaya A, Wesonga H, Thiaucourt F. Use of long-acting tetracyclines for CBPP- preliminary results. Report of the Third Meeting of the FAO/OIE/OAU-IBAR Consultative Group on CBPP, Rome 2003 2004; pp. 112-3.

Huebschle OJ, Ayling RD, Godinho K, et al. Danofloxacin (Advocin) reduces the spread of contagious bovine pleuropneumonia to healthy in-contact cattle. Res Vet Sci 2006; 81(3): 304-9.

Nicholas RAJ, Ayling RD, Tjipura-Zaire G, Rowan T. Treatment of contagious bovine pleuropneumonia. vol. 171. London: BMJ Publishing Group 2012.

Waite ER, March JB. Capsular polysaccharide conjugate vaccines against contagious bovine pleuropneumonia: Immune responses and protection in mice. J Comp Pathol 2002; 126(2-3): 171-82.

March JB, Jepson CD, Clark JR, Totsika M, Calcutt MJ. Phage library screening for the rapid identification and in vivo testing of candidate genes for a DNA vaccine against Mycoplasma mycoides subsp. mycoides small colony biotype. Infect Immun 2006; 74(1): 167-74.

[18] Bischof DF, Janis C, Vilei EM, Bertoni G, Frey J. Cytotoxicity of Mycoplasma mycoides subsp. mycoides small colony type to bovine epithelial cells. Infect Immun 2008; 76(1): 263-9.

[19] Jungi TW, Krampe M, Sileghem M, Griot C, Nicolet J. Differential and strain-specific triggering of bovine alveolar macrophage effector functions by mycoplasmas. Microb Pathog 1996; 21(6): 487-98.

[20] Niang M, Diallo M, Cisse O, et al. Pulmonary and serum antibody responses elicited in zebu cattle experimentally infected with Mycoplasma mycoides subsp. mycoides SC by contact exposure. Vet Res 2006; 37(5): 733-44.

[21] Dedieu L, Balcer-Rodrigues V, Yaya A, et al. Gamma interferonproducing CD4 T-cells correlate with resistance to Mycoplasma mycoides subsp mycoides SC infection in cattle. Vet Immunol Immunopathol 2005; 107(3-4): 217-33.

[22] Abdo el M, Nicolet J, Miserez R, et al. Humoral and bronchial immune responses in cattle experimentally infected with Mycoplasma mycoides subsp. mycoides small colony type. Vet Microbiol 1998; 59(2-3): 109-22.

[23] Masiga WN. Comparative susceptibility of Bos indicus and Bos taurus to contagious bovine pleuropneumonia, and the efficacy of the T1 broth culture vaccine. Vet Rec 1972; 90(18): 499-502.

[24] Windsor RS, Masiga WN. Investigations into the role of carrier animals in the spread of contagious bovine pleuropneumonia. Res Vet Sci 1977; 23(2): 224-9.

[25] Windsor RS, Masiga WN, Read WC. The efficacy of T strain broth vaccine against contagious bovine pleuropneumonia: in-contact trials carried out two years after primary vaccination. Vet Rec 1972; 90(1): 2-5.

[26] Nkando IG, Wesonga HO, Kuria JKN, McKeever D. Assessing the effectiveness of intubation as a challenge model in contagious bovine pleuropneumonia vaccine experiments. Trop Anim Health Prod 2010; 42(8): 1743-7.

[27] Abusugra I, Wolf G, Bolske G, Thiaucourt F, Morein B. ISCOM vaccine against contagious bovine pleuropneumonia (CBPP). 1. 
Biochemical and immunological characterization. Vet Immunol Immunopathol 1997; 59(1-2): 31-48.

[28] Gray MA, Simam P, Smith GR. Observations on experimental inactivated vaccines for contagious bovine pleuropneumonia. J Hyg (Lond) 1986; 97(2): 305-15

[29] Thiaucourt F, Aboubakar Y, Wesonga H, Manso-Silvan L, Blanchard A. Contagious bovine pleuropneumonia vaccines and control strategies: recent data. Dev Biol (Basel) 2004; 119: 99-111.

[30] Gorton TS, Barnett MM, Gull T, et al. Development of real-time diagnostic assays specific for Mycoplasma mycoides subspecies mycoides Small Colony. Vet Microbiol 2005; 111(1-2): 51-8.

[31] Barber TL, Stone SS, Delay PD. Antibody in Cattle Experimentally Infected with Contagious Bovine Pleuropneumonia. Infect Immun 1970; 2(5): 617-22.

[32] Scacchia M, Tjipura-Zaire G, Lelli R, Sacchini F, Pini A. Contagious bovine pleuropneumonia: humoral and pathological events in cattle infected by endotracheal intubation or by exposure to infected animals. Vet Ital 2011; 47(4): 407-13.

[33] Davies G, Masiga WN, Shifrine M, Read WCS. The efficacy of T1 strain broth vaccine against contagious bovine pleuropneumonai: preliminary in-contact trials. Vet Rec $1968 ; 83$ : 239-44.

[34] Brown RD. Endobronchial inoculation of cattle with various strains of Mycoplasma mycoides and the effects of stress. Res Vet Sci 1964; 5: 393-404.

[35] Rottem S. Invasion of mycoplasmas into and fusion with host cells. In: Razin S, Herrmann R, Eds. Molecular Biology and Pathogenicity of Mycoplasmas. New York: Kluwer Academic/ Plenum Publishers 2002; pp. 391-402.

[36] Giron JA, Lange M, Baseman JB. Adherence, fibronectin binding, and induction of cytoskeleton reorganization in cultured human cells by Mycoplasma penetrans. Infect Immun 1996; 64(1): 197208.

[37] Andreev J, Borovsky Z, Rosenshine I, Rottem S. Invasion of HeLa cells by Mycoplasma penetrans and the induction of tyrosine phosphorylation of a $145-\mathrm{kDa}$ host cell protein. FEMS Microbiol Lett 1995; 132(3): 189-94.
[38] Tarshis M, Salman M, Rottem S. Cholesterol is required for the fusion of single unilamellar vesicles with $M$. capricolum. Biophys J 1993; 64: 709-15.

[39] Thomas A, Dizier I, Trolin A, Mainil J, Linden A. Comparison of sampling procedures for isolating pulmonary mycoplasmas in cattle. Vet Res Commun 2002; 26(5): 333-9.

[40] Schubert E, Sachse K, Jores J, Heller M. Serological testing of cattle experimentally infected with Mycoplasma mycoides subsp mycoides Small Colony using four different tests reveals a variety of seroconversion patterns. BMC Vet Res 2011; 7(72): 1-11.

[41] Marobela-Raborokgwe C, Nicholas R, Ayling R, Bashiruddin JB. Comparison of complement fixation test, immunoblotting, indirect ELISA, and competitive ELISA for detecting antibodies to Mycoplasma mycoides subspecies mycoides small colony (SC) in naturally infected cattle from the 1995 outbreak in Botswana. Onderstepoort J Vet 2003; 70(1): 21-7.

[42] Muuka G, Hang'ombe BM, Nalubamba KS, et al. Comparison of complement fixation test, competitive ELISA and LppQ ELISA with post-mortem findings in the diagnosis of contagious bovine pleuropneumonia (CBPP). Trop Anim Health Prod 2011; 43(5): 1057-62.

[43] Bellini S, Giovannini A, di Francesco C, Tittarelli M, Caporale V. Sensitivity and specificity of serological and bacteriological tests for contagious bovine pleuropneumonia. Rev Sci Tech 1998; 17(3): 654-9.

[44] Nicholas RA, Santini FG, Clark KM, et al. A comparison of serological tests and gross lung pathology for detecting contagious bovine pleuropneumonia in two groups of Italian cattle. Vet Rec 1996; 139(4): 89-93.

[45] Nicholas RA, Ayling RD. Mycoplasma bovis: disease, diagnosis, and control. Res Vet Sci 2003; 74(2): 105-12.

[46] Feenstra A, Bisgaard Madsen E, Friis NF, Meyling A, Ahrens P. A field study of Mycoplasma bovis infection in cattle. Zentralbl Veterinarmed B 1991; 38(3): 195-202.

[47] Gagea MI, Bateman KG, Shanahan RA, et al. Naturally occurring Mycoplasma bovis-associated pneumonia and polyarthritis in feedlot beef calves. J Vet Diagn Invest 2006; 18(1): 29-40. 